\title{
Forschungsdesign zur empirischen Untersuchung
}

\subsection{Gütekriterien}

Bevor die Ergebnisse der qualitativen Feldforschung vorgestellt werden, gilt es zunächst, das Forschungsfeld und das Forschungsdesign zu beschreiben, um damit die gebotene Transparenz im Forschungsprozess zu erreichen. Die besondere Herausforderung besteht darin, dass sich klassische Gütekriterien der quantitativen Sozialforschung, wie Reliabilität, Validität und Objektivität, nicht ohne Weiteres auf das Forschungssetting einer qualitativen Untersuchung übertragen lassen. Strategien zur Überprüfung der klassischen Gütekriterien, wie Paralleltestverfahren oder Split-half-Methoden, lassen sich aufgrund der geringen Materialstichprobe kaum anwenden (Mayring 2015, 124). In Bezug auf die Reliabilität soll dennoch in Anlehnung an Uwe Flick (2019) durch eine doppelte Explikation ein möglichst hohes $\mathrm{Maß}$ an Überprüfbarkeit hergestellt werden. Dafür wird einerseits das Zustandekommen der Daten unter Einbeziehung der Auswahlkriterien für das Sampling expliziert, andererseits soll sich die „Reliabilität durch die reflexive Dokumentation des Forschungsprozesses erhöhen" (Flick 2019, 492). Dies soll durch die Beschreibung und Begründung des methodischen Vorgehens (Abschnitt 3.3) und dabei explizit durch die Offenlegung des Interview- und des Kodierleitfadens (siehe Anhang 1) gelingen. Ein mögliches Verfahren zur Erhöhung der Reliabilität ist außerdem die Intercoder-Reliabilität. Gemeint ist damit das mehrfache Kodieren gleicher Textstellen durch unterschiedliche Forscher*innen. Es ist allerdings darauf hinzuweisen, dass dies für Einzelforscher*innen kaum realisierbar ist. Außerdem kann damit Reliabilität nur

Elektronisches Zusatzmaterial Die elektronische Version dieses Kapitels enthält Zusatzmaterial, das berechtigten Benutzern zur Verfügung steht https://doi.org/10.1007/978-3-658-35412-1_3. 
bedingt hergestellt werden, weil dabei zwar das Kodierverfahren selbst in Ansätzen auf Reliabilität überprüft werden kann, für die Erstellung und Überprüfung eines deduktiv und induktiv entwickelten Kodierleitfadens ist dieses Verfahren aber kaum geeignet.

An die Bildung eines Kategoriensystems lässt sich kein Anspruch auf Übereinstimmung stellen. Wenn mehrere Personen auf der Basis des gleichen Materials Kategorien bilden, ist keine perfekte Übereinstimmung zu postulieren und diese lässt sich auch durch das beste Training wohl nicht erreichen. (Kuckartz 2016, 206)

Außerdem verweist Philipp Mayring mit Bezug auf Jürgen Ritsert (1972) darauf, dass dieses Verfahren nur bei sehr einfachen Analysen im Kontext qualitativer Forschung überhaupt zur Anwendung kommen kann (Mayring 2015, 124). Um dennoch dem Anspruch der Reliabilität, zumindest im Rahmen der Möglichkeiten eines Einzelforschungsprojektes, in Ansätzen gerecht werden zu können, wurden Zwischenschritte des Kodierverfahrens anhand konkreter Beispiele aus dem zur Verfügung stehenden Material immer wieder in Forschungskolloquien zur Diskussion gestellt und die Rückmeldung der Peers berücksichtigt. Da die klassischen Gütekriterien quantitativer Forschung für die qualitative Forschung nur bedingt Anwendung finden können, soll damit dem Vorschlag Uwe Flicks für alternative Kriterien entsprochen werden. Mit dem Auditing (Flick 2019, 501 f.), dem Herstellen einer nachvollziehbaren prozeduralen Verlässlichkeit soll durch eine Offenheit unter anderem bezüglich des Samplings, der Kategorienstruktur und des methodischen Zugangs der Forschungsprozess möglichst transparent dargestellt werden. Außerdem wurde das Material in einem mehrstufigen Verfahren analysiert. Dabei konnte in Ansätzen das Prinzip der Intracoderreliabilität greifen: die erneute Analyse des Materials bzw. relevanter Ausschnitte in zwei zeitlich voneinander getrennten Arbeitsphasen (Mayring 2015, 124). Insgesamt soll damit das Vertrauen in die empirischen Ergebnisse der Untersuchung gestärkt und die daraus gezogenen Erkenntnisse damit fundiert begründet werden.

Qualitative Forschung muss sich durch Transparenz in Bezug auf die Phasen des Forschungsprozesses auszeichnen: Vorarbeiten (Theorie, Auswahl des Samplings usw.), Erhebungsphase (Interviewleitfaden, Art der Interviewführung, Transkriptionsregeln usw.) und Analyse des Materials (Methodischer Zugang, Kodierverfahren, computergestützte Auswertungstechniken usw.), Interpretation der Ergebnisse und daraus abgeleitete Implikationen. Der regelgeleitete, prozedurale Charakter der qualitativen Inhaltsanalyse erfordert daher einen besonderen Zugang zur internen und externen Studiengüte. Udo Kuckartz schlägt eine Reihe von Fragen vor, die zur Sicherung der Studiengüte dienen sollen. Diese Fragen 
beziehen sich auf die Datenerfassung (Dokumentation der Daten, Transkriptionsregeln usw.) sowie auf die Durchführung der qualitativen Inhaltsanalyse (gewählter methodischer Zugang, Kodierleitfaden, Umgang mit Originalzitaten usw.). Die mit diesen Fragen verbundene Überprüfbarkeit der Studiengüte „fokussier[t] den prozeduralen Aspekt des Forschungsprozesses und weniger statische Kriterien wie sie in der quantitativen Forschung bspw. in Form von Koeffizienten der Intercoder-Reliabilität berechnet werden“ (Kuckartz 2016, 205). Entsprechend der Kriterien, die Udo Kuckartz für die Erfüllung der Studiengüte vorschlägt, wird im Folgenden - soweit unter Wahrung der Anonymisierung der Daten möglich - dargestellt, wie sich das Forschungssample zusammensetzt (Abschnitt 3.2). Außerdem werden in Abschnitt 3.3 „Methodisches Vorgehen“ die Datenerhebung (3.3.1) und die Datenauswertung (3.3.2) beschrieben. Der Kodierleitfaden ist dem Anhang dieser Arbeit zu entnehmen.

\subsection{Forschungssample}

Die Auswahl des Forschungssamples wurde auf Grundlage eines Verfahrens entwickelt, das nach Reinders auf dem „Sampling durch Selbstaktivierung“ (Reinders 2012, 119 f.) und dem „Profil-Sampling“ (Reinders 2012, 121 f.) beruht. Vor der Feldforschung wurden, von der Forschungsfrage ausgehend, bereits grundlegende Kriterien für die Auswahl der Interviewpartner*innen festgelegt. Da im Zentrum der Untersuchung die (Bildungs)Erfahrungen von Jugendlichen stehen sollten, die selbstbestimmt und selbstorganisiert politisch partizipieren und sich für dieses Engagement in der Schule oder außerhalb der Schule Freiräume suchen bzw. schaffen, war das Sample schon von Beginn an eingeschränkt. Für diese Untersuchung sollten demnach keine Schüler*innen befragt werden, die ausschließlich Erfahrungen durch institutionalisierte Mitwirkungsformate wie die Schüler*innenvertretung gesammelt haben. Auch Kinder und Jugendliche, die ausschließlich Erfahrungen mit außerschulischem politischem Engagement in strukturierten Settings, die den Definitionen konventioneller Partizipationsformen entsprechen, sollten für diese Studie nicht berücksichtigt werden. Deshalb wurden keine Interviews mit Schüler*innen geführt, deren politisches Engagement hauptsächlich auf der Mitwirkung in partei- oder gewerkschaftsnahen Jugendverbänden beruht oder die sich in etablierten Nichtregierungsorganisationen engagieren. Im Fokus stehen die Erfahrungen junger Menschen in Selbstbestimmung und Selbstorganisation. 
Ein weiteres vor Beginn der Feldforschung festgelegtes Kriterium war die Gebundenheit des politischen Engagements an grundlegende Werte und Prinzipien unserer demokratischen Gesellschaft. Ausgangspunkt des eigenen politischen Handelns muss demnach die Anerkennung bzw. Verteidigung von Grund- und Menschenrechten sein. Aus diesem Grund wurden rechtsextreme Jugendgruppen, wie beispielsweise die Identitäre Bewegung, schon im Vorfeld für das Forschungssample ausgeschlossen. Diese durchaus einschränkenden Kriterien für die Vorauswahl des Forschungssamples sind darauf zurückzuführen, dass zu den Zielen der vorliegenden Studie nicht nur die Rekonstruktion politischer (Selbst)Bildungserfahrung zählt, sondern die Forschungsergebnisse auch als Ausgangspunkt für sich daran anschließende Überlegungen zur Einbindung von politischen Aktionen/politischen Projekte in den Schulalltag dienen sollen. Im Sinne des Profil-Samplings lagen, ausgehend von dem ethnografischen Zugang zum Forschungsfeld über forschende Beobachtung bei Protestaktionen, Teilnahme an Demonstrationen und Plenumssitzungen der Jugendprotestgruppen, bereits „Profile der in Frage kommenden Stichprobe“ (Reinders 2012, 121) vor.

Für den Zugang zum Forschungsfeld wurde vor den ersten Interviews zunächst über eine Internetrecherche sowie eine Stichwortsuche in sozialen Netzwerken, wie Facebook, Instagram und Twitter, nach potenziellen Jugendgruppen gesucht, die diesen grundlegenden Kriterien entsprechen. Hierbei ist zu erwähnen, dass der Untersuchungszeitraum zwischen 2016 und 2019 lag. In den ersten Monaten der Feldforschung konnten vor allem Jugendgruppen identifiziert werden, die sich mit Themen wie Migration, Flucht und Vertreibung sowie Integration befassten. Darüber hinaus beschäftigten sich Jugendproteste vor allem im Jahr 2017, anlässlich des G20-Gipfels in Hamburg, mit Fragen der Globalisierung und Herrschaftskritik. Außerdem ist auch das Thema Klima und Umweltschutz schon seit 2017 ein zentrales Anliegen selbstbestimmter Jugendgruppen. Dies ist unter anderem auf die UN-Klimakonferenz in Bonn zurückzuführen. Mit der Gründung der weltweiten Klimaprotestbewegung „Fridays for Future“ im Jahr 2018 und dem großen Zulauf in Deutschland in den ersten Monaten des Jahres 2019 ist das Thema noch stärker in den Fokus gerückt.

Nach einer Desktoprecherche und der Teilnahme an einer Vielzahl von Demonstrationen und weiteren Protestaktionen wurde über verschiedene Wege (persönliche Ansprache, Anschreiben von Jugendgruppen über soziale Netzwerke und via Mail) die Kontaktaufnahme angebahnt. Dies stellte sich als überaus schwierig heraus, da das Misstrauen mir gegenüber zu Beginn sehr groß war. Auch die Autor*innen der ebenfalls qualitativ angelegten Studie „Politisches Engagement und Selbstverständnis linksaffiner Jugendlicher“ (Hillebrand u. a. 2015), die in den Jahren 2012 und 2013 im Feld waren, berichten von einer 
Bandbreite der Haltung zur Beforschung des politischen Engagements zwischen überwiegend offen, skeptisch bis ablehnend.

Die Teilnahmebereitschaft der angefragten Personen war jedoch sehr unterschiedlich. Während bei gemäßigten Gruppen eine überwiegend offene Haltung gegenüber dem Forschungsprojekt vorherrschte, war die Bereitschaft für eine Interviewteilnahme in vielen anderen Gruppen, insbesondere in der linksradikalen Szene, deutlich geringer und bestand bisweilen auch in einer offenen Ablehnung. (Hillebrand u. a. 2015, 49)

Da für die vorliegende Studie keine Interviewpartner*innen in radikalisierten politischen Bewegungen gesucht wurden und keine der politischen Gruppen konspirativ organisiert war, ist es besonders bemerkenswert, dass die anfängliche Skepsis in den Gruppen so ausgeprägt war. Die Sorge vor Überwachung, vor allem durch den Staat, war allgegenwärtig. Obwohl die Gruppen öffentlich tagten und alle Plenumssitzungen, Arbeitstreffen und Protestaktionen allen zugänglich waren, war immer auch eine latente Verunsicherung gegenüber jeder unbekannten Person spürbar. So musste in einigen Plenumssitzungen das Mobiltelefon ausgeschaltet, in anderen Gruppen gar der Akku aus dem Gerät genommen werden. Teilweise musste ich bis zu einem Dutzend Plenumssitzungen und mehrere Demonstrationen besuchen, bis ich ausreichend Vertrauen aufbauen konnte, damit sich aus der Gruppe Interviewpartner*innen fanden, die mit mir sprechen wollten. In einem der Interviews, die für die vorliegende Studie geführt wurden, wird deutlich, auf welchen Erfahrungen diese Skepsis gegenüber staatlichen Institutionen, wie in diesem Fall der Universität, basieren:

[E]s gab auch noch krassere Sachen, die bis hin zu überwachungsstaatlichen Maßnahmen eigentlich gegangen sind, dass man Leute auf der Straße offen angesprochen hat und nach Namen gefragt hat, sogar irgendwie Geld angeboten hat, von Mitarbeitern des Verfassungsschutzes, die sich sogar so vorgestellt haben. Unglaublich. Oder, dass man Leute einschüchtert, dass man Wohnungen durchsuchen wollte, was hier zum Glück noch nie geklappt hat. (FRANZISKA: 873-880)

Die Aussagen lassen sich im Zuge dieser Untersuchung nicht verifizieren, aber tatsächlich berichteten mehrere Jugendliche in unterschiedlichen politischen Gruppen von vergleichbaren Situationen.

Für die empirische Untersuchung zu dieser Arbeit gelang es letztlich dennoch, 17 problemzentrierte leitfadengestützte Interviews (Witzel 1985) zu führen, die zwischen 50 und bis zu 110 Minuten lang waren. Von den 17 Interviews wurden 13 einer qualitativen Inhaltsanalyse unterzogen (ausführlich hierzu 3.3 ,Methodisches Vorgehen“). Eine Gruppe hat nach mehrmonatiger Kontaktanbahnung, 
mehreren Vorgesprächen und der Teilnahme an verschiedenen Plenumssitzungen beschlossen, dass doch niemand aus der Gruppe ein Interview führen wird. Das nach eigenen Aussagen begründete Misstrauen in staatliche Organe vieler Jugendlicher, die für ihr politisches Engagement unkonventionelle und selbstorganisierte Partizipationsformen nutzen, hat den Zugang zum Forschungsfeld erheblich erschwert.

Die interviewten Kinder und Jugendlichen waren im Erhebungszeitraum zwischen 14 und 21 Jahre alt und besuchten zu dieser Zeit verschiedene Schultypen (Berufsschule, Gymnasium und Gesamtschule). Es wurden Personen mit und ohne Migrationsbiografie befragt, aus akademisch und nicht-akademisch geprägten Elternhäusern, die sich in der Schule oder außerhalb individuell und/oder kollektiv in selbstbestimmten Jugendgruppen explizit politisch engagierten. Es wurden Interviews in urbanen Ballungsräumen und Großstädten geführt, aber auch in Kleinstädten und ländlichen Regionen in allen geographischen Teilen Deutschlands.

Im Folgenden werden die Interviewpartner*innen in Kürze vorgestellt. Zum Zwecke der Anonymisierung der Teilnehmenden der Studie wird das Alter nur eingegrenzt und biographische Daten (Name, Wohnort etc.) verändert bzw. verallgemeinert, um eine Rückverfolgung auf die interviewten Personen ausschließen zu können.

EMMA ist zum Zeitpunkt des Interviews zwischen 16 und 18 Jahre alt. Sie ist Schülerin an einer Gesamtschule in einer mittelgroßen Stadt. EMMA hatte private Schicksalsschläge zu überstehen. Vor ihrem politischen Engagement fühlte sich EMMA sozial exkludiert. Ihre formale Bildungslaufbahn war von Frustrationserfahrungen geprägt. EMMA ist in Deutschland geboren. Sie engagiert sich in einer selbstorganisierten politischen Jugendbewegung und setzt sich $u$. a. für den Umweltschutz und gegen Rassismus ein.

ESRA ist zum Zeitpunkt des Interviews zwischen 14 und 16 Jahre alt. Sie ist Schülerin an einem Gymnasium in einer Großstadt. Ihr Elternhaus ist akademisch geprägt. ESRA hat Geschwister. Ihre Eltern leben getrennt. ESRA ist in Deutschland geboren, ihre Familie hat eine Migrationsbiografie und sie hat selbst bereits Rassismuserfahrungen machen müssen. Sie engagiert sich in einer selbstorganisierten politischen Arbeitsgemeinschaft und setzt sich u. a. gegen Rassismus und für Empowermentarbeit für von Rassismus Betroffene ein.

FRANZISKA ist zum Zeitpunkt des Interviews zwischen 18 und 21 Jahre alt. Sie ist Schülerin an einer Berufsschule und macht eine Ausbildung in einem sozialen Beruf. Sie ist seit mehreren Jahren in einer Jugendprotestbewegung aktiv. Ursprünglich ist sie über Bildungsproteste zu der Bewegung gekommen, engagiert 
sich jetzt aber hauptsächlich in den Themenfeldern Klima, Kapitalismus- und Globalisierungskritik sowie Antirassismus.

KATJA ist zum Zeitpunkt des Interviews zwischen 18 und 21 Jahre alt. Sie ist Oberstufenschülerin an einer Gesamtschule in einer mittelgroßen Stadt in Deutschland. Sie engagiert sich in einer Schüler*innengruppe, die Aktionstage gegen Rassismus organisiert. Sie ist aber auch außerschulisch in autonomen politischen Jugendgruppen aktiv.

LEA ist zum Zeitpunkt des Interviews zwischen 14 und 16 Jahre alt. Sie ist Schülerin an einem Gymnasium in einer Großstadt. Ihr Elternhaus ist akademisch geprägt. LEA hat Geschwister und ist in Deutschland geboren. Sie engagiert sich in einer selbstorganisierten politischen Arbeitsgemeinschaft und setzt sich hauptsächlich gegen Rassismus, aber auch gegen Bildungsungerechtigkeit ein. Sie wirkt auch an Schulstreiks mit.

RONJA ist zum Zeitpunkt des Interviews zwischen 16 und 18 Jahre alt. Sie ist Schülerin an einem Gymnasium im ländlichen Raum. Ihr Elternhaus ist akademisch geprägt. RONJA ist in Deutschland geboren. Sie engagiert sich in einer selbstorganisierten politischen Schüler*innen-Initiative und setzt sich hauptsächlich für den Umweltschutz und gegen Rassismus ein. Sie wirkt auch an Schulstreiks mit und ist darüber hinaus eingebunden in einer regionalen außerschulischen Jugendprotestgruppe.

SOPHIE ist zum Zeitpunkt des Interviews zwischen 18 und 21 Jahre alt. Sie ist Schülerin an einer Berufsschule und macht eine Ausbildung in einem handwerklichen Beruf. Sie hat sich lange nicht engagieren wollen, ist jetzt aber in einer politischen Jugendbewegung aktiv, die sich in erster Linie für die Rechte von Geflüchteten einsetzt und gegen Rassismus aktiv ist.

BEN ist zum Zeitpunkt des Interviews zwischen 16 und 18 Jahre alt. Er ist Schüler an einem Gymnasium in einer mittelgroßen Stadt. Er ist in einer Jugendprotestbewegung aktiv, die sich $\mathrm{zu}$ verschiedenen Themenfeldern wie Antirassismus, Antisexismus und Klimawandel engagiert.

HELGE ist zum Zeitpunkt des Interviews zwischen 14 und 16 Jahre alt. Er ist Schüler an einem Gymnasium in einer Großstadt. Er ist in der Schüler*innenvertretung. Er plante politische Aktionstage an seiner Schule und ist darüber hinaus vor allem aktiv in sozialen Netzwerken, um dort politische Diskurse anzuregen und zu begleiten.

JAN ist zum Zeitpunkt des Interviews zwischen 16 und 18 Jahre alt. Er ist Schüler an einem Gymnasium in einer Kleinstadt. Er engagiert sich gegen Rassismus, ist aber vor allem ein Einzelkämpfer, der von sich selbst sagt, dass er sich derzeit in der Phase der Radikalisierung befindet. 
JOHANNES ist zum Zeitpunkt des Interviews zwischen 16 und 18 Jahre alt. Er ist Oberstufenschüler einer Gesamtschule und aktiv in einer politischen Arbeitsgemeinschaft an seiner Schule, die sich gegen Rassismus engagiert. Er hat einen Aktionstag organisiert, ist an seiner Schule darüber hinaus klimapolitisch aktiv und bringt sich außerhalb der Schule in jugendpolitischen Initiativen ein.

LAURENZ ist zum Zeitpunkt des Interviews zwischen 16 und 18 Jahre alt. Er ist Schüler an einem Gymnasium in einer mittelgroßen Stadt. Er ist aktiv in einer Jugendprotestbewegung, die sich zu verschiedenen Themenfeldern engagiert. Für ihn spielen Kapitalismus-, Globalisierungs- und Systemkritik eine wichtige Rolle.

MICHAEL ist zum Zeitpunkt des Interviews zwischen 14 und 16 Jahre alt. Er ist Schüler an einem Gymnasium im ländlichen Raum. Er ist in der Schüler*innenvertretung und hat eine Demonstration gegen Rassismus mit der Schulgemeinschaft maßgeblich mitorganisiert.

\subsection{Methodisches Vorgehen}

\subsubsection{Datenerhebung}

Für die vorliegende qualitative Untersuchung wurden in den Jahren 2017 bis 2019 insgesamt 17 leitfadengestützte problemzentrierte Interviews (Witzel 1985) mit Kindern und Jugendlichen geführt, die sich selbstbestimmt und selbstorganisiert politisch engagieren.

Grundsätzlich zeichnet sich das problemzentrierte Interview durch zwei Kommunikationsstrategien aus. Gerade zu Beginn des Interviews werden vor allem erzählungsgenerierende Techniken eingesetzt, während im weiteren Verlauf verstärkt verständnisgenerierende Techniken genutzt werden. (Fischer u. a. 2016, 23)

Die Interviews wurden mit einem digitalen Aufnahmegeräten aufgezeichnet und vollständig transkribiert (Dresing/Pehl 2010). Sie hatten im Schnitt eine Länge von ca. 70 Minuten, wobei der überwiegende Teil der Interviews mehr als eine Stunde dauerte. Erstellt wurde ein inhaltlich-semantisches Transkript (Dresing/Pehl 2018, 18-23). Folgende Transkriptionsregeln wurden dabei angewandt:

1. Da die inhaltliche Ebene des Interviews im Fokus der Studie steht, wird wörtlich transkribiert und nicht lautsprachlich. Dialekte werden nach Möglichkeit geglättet, also möglichst ins Hochdeutsch übertragen. 
2. Sprache und Interpunktion werden ebenfalls geglättet, d. h. an das Schriftdeutsch angenähert. Die Satzform, bestimmte und unbestimmte Artikel etc. werden auch dann beibehalten, wenn sie Fehler enthalten. Das Hauptaugenmerk liegt darauf, den Inhalt / Gedanken des Sprechers / der Sprecherin möglichst nah am gesprochenen Wort zu transkribieren.

3. Pausen ab drei Sekunden werden durch in Klammern gesetzte Auslassungspunkte markiert. Die Länge der Pause wird durch eine Zahl entsprechend der Sekunden festgehalten. Eine Sprechpause von fünf Sekunden wird demnach wie folgt dokumentiert: (...5...)

4. Besonders hervorgehobene Begriffe werden durch Unterstreichung gekennzeichnet.

5. Sehr lautes Sprechen wird durch Schreiben in GROSSBUCHSTABEN kenntlich gemacht.

6. Zustimmende bzw. bestätigende Lautäußerungen des Interviewers ( $\mathrm{mhm}$, aha etc.) werden nicht mit transkribiert, sofern sie den Redefluss der befragten Person nicht unterbrechen.

7. Einwürfe einer Person werden, sofern der Satz der jeweils anderen Person unterbrochen wird, durch drei Punkte vor und nach der Einlassung gekennzeichnet. Ein Beispiel:

I: Ich würde gerne von dir wissen, welche Erfahrungen du mit Rassismus und Ausgrenzung ...

SI1: ... ständig, in der Bahn...

I: ... in der Schule. Wollte ich eigentlich fragen. Aber berichte doch bitte gern erst mal von deinen Alltagserfahrungen mit Rassismus.

8. Jeder Sprechbeitrag wird als eigener Absatz transkribiert. Wechsel zwischen der interviewten und der interviewenden Person werden durch eine Leerzeile verdeutlicht.

9. Störungen werden unter Angabe der Ursache in eckigen Klammern notiert, z. B. [Handy klingelt].

10. Indirektes Sprechen wird mit folgenden Zeichen markiert $>>$ Beispieltext $<<$. Ein Beispiel:

P2: Uns wird immer wieder etwas von Mitschülerinnen und Mitschülern unterstellt. Sie sagen zum Beispiel immer wieder $>>$ Ihr macht da nur mit, um euch einzuschleimen $<<$.

11. Nonverbale Aktivitäten und Äußerungen der befragten wie auch der interviewenden Person werden in einfachen Klammern notiert, z. B. (lacht), (seufzt) und Ähnliches.

12. Unverständliche Wörter werden durch (unv.) kenntlich gemacht. 
13. Alle Angaben, die einen Rückschluss auf eine befragte Person erlauben, werden anonymisiert.

Das vollständige Materialkorpus bestand insgesamt aus über 1.000 Minuten digitalen Audiomitschnitten, einem Transkript von mehr als einer Million Zeichen und damit knapp 400 Seiten Datenmaterial. Ausgewertet wurden mit 13 Interviews ca. $80 \%$ des Datenmaterials. Zwei Interviews wurden nachträglich zurückgezogen. Das Material wurde demzufolge vernichtet. Zwei Interviews wurden mit Jugendlichen geführt, die in einer Protestbewegung aktiv waren, aus der bereits ein Proband interviewt wurde. Aufgrund der gehäuft ähnlichen/vergleichbaren Aussagen wurde die Einbeziehung der Interviews für nicht notwendig bzw. nicht zielführend erachtet.

Im Folgenden werden wesentliche Merkmale des Interviewleitfadens vorgestellt. Dabei wird auch auf die Kriterien eingegangen, die grundlegend für die Entwicklung des Leitfadens waren. Ausgangspunkt der Überlegungen sind die Prinzipien der qualitativen Sozialforschung: Offenheit, Prozesshaftigkeit und Kommunikativität (Reinders 2012, 128). Das Forschungsprinzip orientiert sich an der rekonstruktiven Sozialforschung (Bohnsack 2014). Die gewählten Fragen sind daher grundsätzlich offene Fragen, die es den interviewten Personen ermöglichen, frei und unvoreingenommen mit eigenen Worten zu antworten (Bohnsack 2014, 22). Um die Offenheit der Fragen zu gewährleisten und zugleich die notwendige Struktur für den Leitfaden zu entwickeln, empfiehlt Helfferich $(2011,182)$ ein Vorgehen, das mit „SPSS bei der Leitfadenerstellung“ abgekürzt wird. „Es dient gleichzeitig der Vergegenwärtigung und dem Explizieren des eigenen theoretischen Vorwissens und der impliziten Erwartungen an die von den Interviewten zu produzierenden Erzählungen.“ (Helfferich 2011, 182) SPSS steht für vier Schritte bei der Entwicklung des Leitfadens.

Im ersten Schritt „Sammeln“ werden alle potenziell relevanten Fragen, die sich vor allem deduktiv aus den Überlegungen zur Recherche des Forschungsstandes ergeben, zusammengestellt. Für die vorliegende Arbeit bezog sich dies zunächst auf Fragen zu den Vorstellungswelten der Befragten in Bezug auf die Konzepte „Politik“ und „Demokratie“. Ausgehend von den Erfahrungsberichten und den Informationen über die individuellen Motive in Bezug auf die eigene politische Teilhabe wurden darüber hinaus Fragen formuliert, die die jeweiligen Handlungserfahrungen mit den Kategorien des politischen Lernens zusammenführen. Die Fragensammlung erstreckte sich daher vor allem auf die Ebene des sozialwissenschaftlichen Analysierens und Denkens, der politischen Orientierung, der Perspektivenwahrnehmung, der politischen Urteilsbildung und der Handlungskompetenz. 
Im zweiten Schritt „Prüfen“ gilt es, die Sammlung an Fragen auf einen Kern an zentralen Fragen zu reduzieren und zugleich sicherzustellen, dass dabei die wesentlichen Impulse für das Interview bestehen bleiben.

Im abschließenden dritten Schritt werden die Fragen systematisch sortiert (Helfferich 2011, 182-185). Für die Sortierung des Fragebogens empfiehlt Reinders (2012, 133-138) den Ablauf in drei Phasen zu unterteilen: Warm-Up, Hauptphase und Ausklang. Für die Warm-Up-Phase ist für die vorliegende Studie, anders als in der Literatur zumeist empfohlen, kein möglichst niedrigschwelliger Zugang gewählt worden. Die erste Frage ist eher als Ad-Hoc-Einstieg gedacht. Ziel war es, ohne die Eindrücke der nachfolgenden Frage zu den individuellen Partizipationserfahrungen, die Vorstellungen der Befragten zu Politik und Demokratie zu eruieren, weil davon ausgegangen werden konnte, dass eine Frage nach ihrem Verständnis dieser Begriffe bei politisch interessierten Jugendlichen einen erzählauffordernden Impuls darstellt. Daher war der erste Impuls die Bitte zu beschreiben, was die befragte Person mit dem Begriff „Politik“ verbindet. Aufgrund der Besonderheit des Samples war davon auszugehen, dass alle Befragten hierzu klare Vorstellungen formulieren können. Dies bestätigte sich auch in einem Pre-Test des Leitfadens. In der Hauptphase wurden die Fragen dann in den Themenblöcken politische Analyse und Orientierung, politisches Urteil und Kritik sowie die Beschreibung der eigenen Handlungserfahrungen strukturiert.

Im letzten Schritt, dem „Subsumieren“, wurde für jedes Themenfeld eine möglichst einfache Erzählaufforderung formuliert, die möglichst offen die Befragten dazu anregen sollte, von gegenwärtigen und zurückliegenden politischen Partizipationserfahrungen zu berichten und diese zu reflektieren (Helfferich 2011, 185). Folgende Erzählaufforderungen wurden im Leitfaden formuliert (angelehnt an: Reinders 2012, 138):

- Ad-Hoc-Einstieg: Was verbindest du mit dem Begriff „Politik“?

- Erster Impuls für den narrativen Teil: Berichte mir doch bitte zunächst von deinem politischen Engagement.

- Analyse und Orientierung: Erzähl mir doch bitte davon, wie ihr euch in der Gruppe Informationen über die Themen beschafft, die euch interessieren.

- Urteil und Kritik: Wie findet ihr in eurer Gruppe eine gemeinsame Position bei konfliktreichen Themen?

- Handlungserfahrung: Erzähl mir bitte etwas über die unterschiedlichen politischen Aktionsformen, die du in deiner aktiven Zeit kennengelernt hast.

Der Leitfaden beinhaltet neben den erzählauffordernden und textgenerierenden Fragen auch aufrechterhaltende Fragen (Dresing/Pehl 2018, 10). Darüber hinaus 
wurde darauf geachtet, dass die Fragen und geplanten Themenfelder ,nicht zu abrupten Sprüngen und Themenwechseln“ (Helfferich 2011, 180) führten. Am Ende des Interviews wurden die Jugendlichen selbst als Expert*innen wahrgenommen und danach befragt, was sie sich von der Institution Schule wünschen würden. Außerdem wurde den Interviewten abschließend die Möglichkeit gegeben, ,eigene Relevanzen zu setzen und den Interviewverlauf zu kommentieren“ (Helfferich 2011, 181).

Grundsätzlich galt für die Interviews die Maßgabe, den Befragten zu ermöglichen das zentrale Thema des Interviews in ihrer „eigenen Sprache, in ihrem Symbolsystem und innerhalb ihres Relevanzrahmens entfalten zu lassen" (Bohnsack 2014, 22), um zu verhindern, dass einzelnen Äußerungen Bedeutungen beigemessen werden, die ihnen nicht zukommen. Dieser Grundsatz ist auch für die Datenauswertung von Bedeutung. Einzelaussagen gilt es demnach, immer auch im Gesamtkontext des Gesagten einzuordnen.

\subsubsection{Datenauswertung}

Die Aufbereitung und Auswertung des im Zuge der Feldforschung erhobenen Datenmaterials richtete sich nach den Prinzipien der qualitativen Sozialforschung (Flick 2019). Wenngleich sich der methodische Zugang vor allem an den Überlegungen zur rekonstruktiven Sozialforschung (Bohnsack 2014) orientiert, wird bezüglich der Instrumentarien zur Strukturierung, der Analyse und Interpretation des Datenmaterials weder auf die dokumentarische Methode, noch auf die objektive Hermeneutik zurückgegriffen, sondern auf das Verfahren der qualitativen Inhaltsanalyse.

Die qualitative Inhaltsanalyse zeichnet sich dadurch aus, dass das Verfahren kategorienbasiert, komprimierend und resümierend, „,mit der Intention der Zusammenfassung - und auch Reduktion von Komplexität - angewandt“ (Kuckartz 2016, 52) wird. Es handelt sich demnach um ein systematisches, regelgeleitetes Verfahren (Kuckartz 2016, 53). Das systematische Vorgehen zeigt sich darin, dass die Analyse „nach expliziten Regeln abläuft“ (Mayring 2015, 12) und damit einen möglichst hohen Grad an intersubjektiver Überprüfbarkeit ermöglicht.

Für die vorliegende Studie wurde sich an den von Udo Kuckartz vorgeschlagenen Schritten für die inhaltlich strukturierende Inhaltsanalyse (Kuckartz 2016, 100-111) und der strukturierenden Inhaltsanalyse nach Philipp Mayring (2015, 97-114) orientiert. 
Ziel inhaltlicher Strukturierung ist es, bestimmte Themen, Inhalte, Aspekte aus dem Material herauszufiltern und zusammenzufassen. Welche Inhalte aus dem Material extrahiert werden sollen, wird durch theoriegeleitet entwickelte Kategorien und (sofern notwendig) Unterkategorien bezeichnet. (Mayring 2015, 103)

Dabei wird im Anschluss an die initiierende Textarbeit, dem theoriegeleiteten Definieren von Hauptkategorien (deduktiv) sowie der darauf aufbauenden Formulierung des deduktiven Kodierleitfadens entlang dieser Hauptkategorien das Material kodiert. „In der nächsten Phase werden die induktiven Kategorien am Material weiterentwickelt und ausdifferenziert. Das gesamte Datenmaterial wird anschließend in einem zweiten Materialdurchlauf erneut codiert, im Folgenden kategorienbasiert ausgewertet und für den zu schreibenden Forschungsbericht aufbereitet.“ (Kuckartz 2016, 97)

Das Vorgehen wurde auf Grundlage der methodischen Konzeptionen von Kuckartz $(2016,100-111)$ und Mayring $(2015,97-114)$ wie folgt adaptiert:

\section{Vorgelagerte Phasen}

Phase 1: Theoretische Begründung der Fragestellung

Phase 2: Fachliche Klärung bzw. Analyse des Forschungsstandes

Phase 3: Erste Strukturierung der thematischen Felder

Phase 4: Feldforschung: Erhebung des Materials

Phase 5: Transkription

\section{Phase der Strukturierung des Materials}

Phase 6: Initiierende Textarbeit, Markieren \& Kommentieren wichtiger Textstellen

Phase 7: Definieren von thematischen Kategorien (theoriegeleitet)

Phase 8: Formulierung eines Kodierleitfadens für den ersten Materialdurchlauf

Phase 9: Erster Kodierprozess: Kodieren des gesamten Materials mit den deduktiv entwickelten Hauptkategorien - parallel: induktives Bestimmen zusätzlicher Kategorien

Phase 10: Nach erstem Materialdurchlauf: Überarbeitung / Revision des Kodierleitfadens

Phase 11: Zweiter Kodierprozess: Erneutes Kodieren des kompletten Materials mit dem deduktiv und induktiv entwickelten Kodierleitfaden

\section{Phase der Analyse, Auswertung und Zusammenfassung}

Phase 12: Kategorienbasierte Auswertung und Zusammenfassung der Subkategorien sowie Gewichtung und Auswahl „repräsentativer“ Textstellen 
Phase 13: Zusammenhänge der Subkategorien innerhalb einer Hauptkategorie herausarbeiten

Phase 14: Abschließende Zusammenfassung und Darstellung der Analyseergebnisse unter Einbeziehung ,repräsentativer“ Textstellen

Die Strukturierung des Materials wurde weitestgehend mit dem von Udo Kuckartz entwickelten Software-Programm MAXQDA vorgenommen. Die Aussagen der interviewten Personen wurden in einem mehrstufigen Prozess (deduktive und induktive Kategorienbildung) kodiert, kommentiert und in ihrer Bedeutung für die Arbeit gewichtet. Dafür wurde das Material in Kodiereinheiten gegliedert, die einen Umfang von einer Wortgruppe bis zu mehreren Absätzen umfassen können. Sofern die (Nach)Fragen für das Verständnis der Sinneinheit notwendig war, wurden diese ebenfalls kodiert. „Beim Zuordnen der Kategorien gilt es, ein gutes $\mathrm{Maß}$ zu finden, wie viel Text um die relevante Information herum mitcodiert wird. Wichtigstes Kriterium ist, dass die Textstelle ohne den sie umgebenden Text für sich allein ausreichend verständlich ist." (Kuckartz 2016, 104)

Die Hauptkategorien entsprechen einer thematischen Sortierung, die sich theoriegeleitet an den Diskursen zum politischen Lernen aus der politischen Bildung ableiten (siehe Abschnitt 2.2 Politische Bildung - Zugänge, Kompetenzen und Erfahrungsräume): „Analysefähigkeit und politische Orientierung“, „Urteil und Kritik“, „Partizipation und Konfliktfähigkeit (Individuelle und kollektive Handlungsfähigkeit)“ sowie „Politisches Selbstkonzept (Agency)“. Darüber hinaus bilden „Motivation für politische Aktion“ und „Politik- / Demokratieverständnis“ weitere Hauptkategorien, die sich aus dem gesprächsanleitenden Einstieg in die Interviews ergeben. Für die genauere Strukturierung wurden wenige zusätzliche Unterkategorien deduktiv und theoriegeleitet festgelegt. Außerdem ergaben sich weitere induktiv bestimmte Haupt- und Unterkategorien. Die deduktiv bestimmten Kategorien werden im Folgenden fett dargestellt, die induktiv bestimmten Kategorien kursiv:

HK1 Motivation für politische Partizipation

HKl UKl Bedürfnis, aktiv zu werden

HK1 UK2 Gruppendynamik / Gruppenprozesse (sozialer Faktor)

HK1 UK3 Unzufriedenheit mit Verhältnissen

HK1 UK4 Sozialisation

HK2Politik- / Demokratieverständnis

HK2 UK1 Politik(er*innen)verdrossenheit

HK2 UK2 Beschreibung des Status Quo

HK2 UK2.1 Komplexität / Prozesshaftigkeit 
HK2 UK2.2 Allgemeines Verständnis (Werte, Prinzipien)

HK2 UK2.3 Demokratie als Lebensform

HK2 UK2.4 Demokratie als Gesellschaftsform

HK2 UK2.5 Demokratie als Herrschaftsform

HK2 UK3 Utopie / Idealvorstellung

HK2 UK3.1 Grundsätze

HK2 UK3.2 Strukturelle Überlegungen

HK3 Analysefähigkeit und politische Orientierung

HK3 UK1 Offenheit im politischen Diskurs

HK3 UK2 Quellenanalyse

HK3 UK3 Konflikt- und Gesellschaftsanalyse

HK3 UK4 Reflexion der eigenen Konfliktfähigkeit

HK4 Urteil und Kritik

HK4 UK1 Urteilsbildung durch Partizipation

HK4 UK2 Wert der Meinungsbildung

HK4 UK3 Kritik / Gesellschaftskritik

HK4 UK4 Reflexion der eigenen Urteilsfähigkeit

HK5 Individuelle und kollektive Handlungsfähigkeit

HK5 UK1 Öffentlichkeit herstellen

HK5 UK2 Soziales Engagement

HK5 UK3 Politische Partizipation

HK5 UK3.1 legal (innerhalb / außerhalb der Schule)

HK5 UK3.2 illegal / ziviler Ungehorsam

HK5 UK4 Konfliktfähigkeit

HK5 UK5 Reflexion des eigenen Handelns

HK6Agency / Politisches Selbstkonzept

HK6 UK1 Frustration und Anerkennung

HK6 UK2 Wir-Ihr-Konstruktionen

HK6 UK3 Politisierung und Persönlichkeit

Das Material wurde mithilfe der qualitativen Inhaltsanalyse anhand dieses deduktiv-induktiven Kategorienleitfadens in dem oben beschriebenen mehrstufigen Analyseverfahren strukturiert. Der methodische Zugang wurde gewählt, um anhand der Strukturierung der Aussagen verschiedene Ansätze politischer (Selbst)Bildung auch unter Berücksichtigung fachwissenschaftlicher und fachdidaktischer Expertise rekonstruieren zu können.

Im empirischen Teil dieser Arbeit werden die Erkenntnisse des Analyseprozesses zusammengefasst. Dabei werden in Anlehnung an Kuckartz nicht nur „Techniken selektiver Plausibilisierung angewendet [,sondern es wird] auch auf 
Gegenbeispiele und Widersprüche hingewiesen“ (Kuckartz 2016, 205). Dafür werden repräsentative bzw. herausragende Aussagen der Interviewten wörtlich zur Illustration angeführt. Andere beispielhafte Erfahrungsberichte werde paraphrasiert. Vergleichbare Erfahrungsberichte werden zusammengefasst oder exemplarisch vorgestellt. Hervorgehoben werden abweichende Fälle und Ausnahmefälle.

Im folgenden Kapitel 4 „Politische Aktion als Bildungserfahrung. Empirische Analyse" werden die Ergebnisse der empirischen Analyse zusammengefasst. Einführend werden in Abschnitt 4.1 anhand zweier Fallbeispiele die vielfältigen Demokratie- und Politikvorstellungen der interviewten Jugendlichen vorgestellt. Dieses Kapitel verfolgt nicht den Anspruch, im Sinne einer politikdidaktischen Rekonstruktion (Heidemeyer/Lange 2010; Kattmann 2007; Vajen u. a. 2021) alle Facetten der subjektiven Vorstellungen und Sinnbilder der Jugendlichen herauszuarbeiten. Vielmehr soll damit ein Einblick in die komplexen Deutungsmuster der politisch aktiven Jugendlichen ermöglicht werden. Daran anschließend werden in Abschnitt 4.2 die motivationalen Faktoren für die Bereitschaft zur politischen Aktion herausgearbeitet. An diese beiden einführenden Kapitel, die hauptsächlich auf induktiv gewonnenen Auswertungskategorien basieren, schließt das Abschnitt 4.3 „Rekonstruktion politischer (Selbst)Bildungserfahrungen“ an. Hier werden die Erfahrungsberichte der Jugendlichen unter anderem auf Grundlage der in Abschnitt 2.2 zusammengefassten Zugänge, Kompetenzen und Erfahrungsräume politischer Bildung rekonstruiert. Bewusst stehen dabei die exemplarischen Aussagen der Interviewten für sich und werden nur durch strukturierende Kommentierungen gerahmt. Eine wissenschaftliche Diskussion und Einordnung der Ergebnisse erfolgt bewusst erst am Ende des Kapitels, um nicht Gefahr zu laufen, die Rekonstruktion der (Selbst)Bildungserfahrungen zu relativieren oder defizitorientiert zu dekonstruieren. 
Open Access Dieses Kapitel wird unter der Creative Commons Namensnennung 4.0 International Lizenz (http://creativecommons.org/licenses/by/4.0/deed.de) veröffentlicht, welche die Nutzung, Vervielfältigung, Bearbeitung, Verbreitung und Wiedergabe in jeglichem Medium und Format erlaubt, sofern Sie den/die ursprünglichen Autor(en) und die Quelle ordnungsgemäß nennen, einen Link zur Creative Commons Lizenz beifügen und angeben, ob Änderungen vorgenommen wurden.

Die in diesem Kapitel enthaltenen Bilder und sonstiges Drittmaterial unterliegen ebenfalls der genannten Creative Commons Lizenz, sofern sich aus der Abbildungslegende nichts anderes ergibt. Sofern das betreffende Material nicht unter der genannten Creative Commons Lizenz steht und die betreffende Handlung nicht nach gesetzlichen Vorschriften erlaubt ist, ist für die oben aufgeführten Weiterverwendungen des Materials die Einwilligung des jeweiligen Rechteinhabers einzuholen. 